\title{
Biased by design? Clinical trials and patient benefit in oncology
}

\author{
Pan Pantziarka*,1,2 (iD), Ciska Verbaanderd ${ }^{1,3}$ \& Lydie Meheus ${ }^{1}$ \\ ${ }^{1}$ The Anticancer Fund, 1853 Strombeek-Bever, Brussels, Belgium \\ ${ }^{2}$ The George Pantziarka TP53 Trust, London, UK \\ ${ }^{3}$ Clinical Pharmacology \& Pharmacotherapy, Department of Pharmaceutical \& Pharmacological Sciences, KU Leuven, Belgium \\ *Author for correspondence: anticancer.org.uk@gmail.com
}

First draft submitted: 20 November 2019; Accepted for publication: 27 November 2019; Published online: 11 December 2019

Keywords: clinical trial design $\bullet$ drug development $\bullet$ drug registration $\bullet$ patient benefit $\bullet$ risks of bias

\section{Pivotal clinical trials: biased?}

A recent study by Naci et al. examined the design characteristics, risks of bias and reporting adequacies of pivotal randomized controlled trials of cancer drugs approved by the EMA in the period 2014-2016 [1]. During this period, 32 new cancer drugs were approved by the EMA on the basis of 54 pivotal trials - of these, $41(76 \%)$ were randomized controlled trials and $13(24 \%)$ were non-randomized or single arm trials. The study reported that $49 \%$ of trials were judged to be at high risk of bias based on aspects of their design, conduct or analysis. Furthermore, only $10(26 \%)$ randomized controlled trials measured overall survival as a primary end point, with the majority of trials evaluating surrogate metrics such as progression free survival or response rates. Furthermore, there were also discrepancies between scientific publications and regulatory documentation, thereby raising concerns of reporting inadequacies and risks of bias in both sources of information. These findings raise serious questions regarding clinical trial design and the drug registration process, particularly with regards to patient benefits.

The problem is not restricted to Europe alone; previous studies have also raised concerns with US FDA approvals. For example, a study that assessed all FDA approvals of novel drugs, across all areas of medicine in the period 2005-2012, reported wide disparities in pivotal trial characteristics across different therapeutic areas [2]. While overall 219 of 448 (48.9\%) pivotal trials included a surrogate end point, for cancer it was 46 of 55 (83.6\%). Pivotal trials in cancer were also less likely to be randomized, double-blinded or to not include a placebo or active comparator. Another notable finding was that most cancer approvals were on the basis of a single pivotal trial, whereas for other therapeutic areas, approvals were based on two to four pivotal trials. A different study examining FDA cancer drug approvals based on response rate showed that many cancer drugs are approved based on low or modest response rates, typically tested in single-arm studies [3].

It could be argued that the surrogate end points used in such pivotal trials are acceptable as they correlate to overall survival and/or quality of life in the long term [4]. However, the data to support such an argument is lacking [5]. A recent systematic review of the association between surrogate end points and overall survival in oncology showed that the vast majority (82\%) are low or moderate in strength [6]. A study of FDA approvals of cancer drugs during 2008-2012, a period in which 67\% of approvals were made on the basis of a surrogate end point, assessed whether subsequent data emerged to show an increase in overall survival [7]. With a median follow-up of 4.4 years, the study reported that of 36 drugs approved with surrogate end points, only five subsequently reported improved overall survival in a randomized controlled trial, 18 had been shown to not improve overall survival in subsequent trials and 13 had not been tested or had not reported overall survival in subsequent trials. Similar results have also been reported for EMA approvals for drugs approved without a survival or quality of life end point [8]. Indeed, equally concerning is the fact that, in many cases, even the surrogate end points in pivotal trials are not subsequently replicated in post-approval studies [9]. 


\section{Clinical benefits}

These concerns regarding trial design and risks of bias relate to the scale of clinical benefits that these newly approved drugs bring to clinical practice. The European Society for Medical Oncology Magnitude of Clinical Benefit Scale (ESMO-MCBS) is a tool used to assess the clinical benefit of novel cancer therapies [10] and has been reported to correlate with clinically relevant survival gains [11]. Using both the ESMO-MCBS and a modified version adapted for Health Technology Assessment (HTA) [12], an analysis of 5 years (2011-2016) of EMA cancer drug approvals showed that 89 and $79 \%$ of therapies did not meet the MCBS or modified threshold respectively [13]. A USA analysis, using the ESMO-MCBS threshold, showed that over the period of 2006-2016, only $43.8 \%$ of randomized controlled trials supporting FDA approvals for new cancer drugs met the MCB threshold [14].

In addition to therapeutic benefit to patients, there are also significant financial implications associated with these trends. Saluja and colleagues assessed trends in costs and clinical benefits of new cancer drugs approved by the FDA in the period 2006-2015 [15]. The study looked at Phase III randomized controlled trials used to support drug approvals. Using both the ESMO-MCBS and the American Society of Oncology Value Framework, it assessed the secular trend of degree of clinical benefit reported and monthly drug pricing. The data showed that whereas the degree of clinical benefit remained static or declined over the study period, drug pricing showed repeated yearly increases, with no correlation to degree of clinical benefit. It is also notable that, in line with the study by Naci et al., only $29 \%$ of included trials had overall survival as the primary end point. Vivot et al. showed similar findings for FDA approvals between 2000 and 2015 for new medicines to treat advanced solid cancer. Only $35 \%$ of evaluable drugs in this study showed meaningful clinical benefit and there was no relation between the price of drugs and their clinical benefit [16].

The picture that emerges from these various studies is a disturbing one - it suggests that drug development, which is generating increasingly expensive new agents, is failing to deliver concomitant improvements in clinical benefit to patients. Can digging into the data shed more light on the underlying causes of this trend?

A recent analysis, which included one of the present authors (P Pantziarka), assessed the relative contributions of academia and industry in supporting trials for treatments included in the National Comprehensive Cancer Network guidelines for non-small-cell lung cancer [17]. The data showed that, of 57 recommended interventions, 39 (68\%) were based on at least one randomized controlled trial. All 17 distinct interventions demonstrated an improvement in overall survival in 26 trials published between 1990 and 2017. Of these, five (29\%) interventions improved overall survival in the curative setting and $12(71 \%)$ improved overall survival in the noncurative setting, with the largest gains in survival being in the curative setting. Of the 26 trials, $12(46 \%)$ were sponsored by academic groups and largely funded from public sources, whereas for the 14 (54\%) industry-sponsored trials, the funding came from industry sources alone. However, whereas the academic trials included a range of nondrug interventions, with more than half $(58 \%)$ in the curative setting, the industry-sponsored trials were weighted heavily $(79 \%)$ toward drug trials in the noncurative setting. This preponderance of trials in noncurative settings is supported in other studies $[8,18,19]$.

\section{Lowering the bar}

It is a truism that people and organizations respond to incentives. In this case, there is a clear signal that the noncurative setting is the more attractive one for industry. The evidentiary bar is lower; approvals can be gained based on nonrandomized trials or progression-free survival or response rate outcomes. A very recent example is the FDA approval of the poly (ADP-ribose) polymerase (PARP) inhibitor niraparib for homologous recombination deficiency-positive advanced ovarian cancer on the basis of a 98-patient single-arm study (NCT02354586) with a primary outcome of overall response rate (24\%) and duration of response (median 8.3 months, all of them partial responses) [20]. The standard development path for industry is to gain a first approval in a single cancer setting and then to extend to other cancers or to an earlier stage of treatment (i.e., from third line to second line to first line or from metastatic to primary disease). Given that early phase trials are usually in the advanced or metastatic setting and that approvals in this setting may be gained with fewer patients or lower levels of evidence, this is an entirely rational strategy.

However, as the study of National Comprehensive Cancer Network non-small-cell lung cancer guidelines showed, greater survival benefits are often achieved using existing drugs and/or nondrug treatments in the curative setting. Randomized clinical trials of such interventions are generally not commercially supported and depend primarily on public or philanthropic sources of funding. Similarly, drug repurposing, a drug development strategy that seeks to use existing licensed drugs in new medical indications, also faces funding challenges. We recently studied the 
potential of drug repurposing in oncology and showed that, of 190 late-stage clinical trials exploring the use of noncancer drugs as cancer therapeutics, less than $4 \%$ had funding from commercial sources [21].

Of course drug development does not happen in a vacuum - payers, policy-makers, patients and others exert influence in an attempt to steer development in ways perceived to be more beneficial to society as a whole. Orphan drug designation, accelerated and conditional approvals - there are many different mechanisms that regulators are using to engineer the incentives for drug development. And yet the problems remain - for example, in the study which assessed post-approval outcomes for drugs approved by the FDA on the basis of surrogate outcomes, 15 of the 36 drugs were accelerated approvals and, of these 15, only one was subsequently shown to deliver improved overall survival [22]. Some have characterized the current situation as incentivizing too many 'me-too' drugs - that is, the development of virtual duplicates of profitable drugs by competing companies - thereby stifling innovation and delivering marginal benefits to patients [23].

\section{Addressing the problems}

Given the situation outlined above, we must ask what measures are required to address the serious issues raised, taking into account both the urgent need for timely access to treatment options for cancer patients and the requirement for robust efficacy data (improved overall survival or quality of life) before medicines can enter the market. Being aware of the dangers of creating perverse incentives or unintended consequences arising from wholesale change, the following may help to address some of the issues:

\section{- Raise the evidentiary bar in pivotal clinical trials}

Because of the weak associations between hard and surrogate end points, we recommend that regulators require overall survival and quality of life as primary end points in pivotal clinical trials wherever feasible. In cases where surrogate end points have demonstrated robust ability to predict meaningful benefits or where it is not possible to measure hard end points in a pivotal trial, for example, for very rare cancers or cancers with no effective standard of care, conditional or accelerated regulatory approval based on surrogate end points could be useful [5]. However, if rigorously designed post-approval studies fail to show significant clinical benefit based on hard end points in the target population, the marketing authorization and/or reimbursement should be revoked.

Similarly, Phase III randomized controlled trials should remain the standard for regulatory approval. However, under exceptional circumstances, it may be appropriate to grant accelerated or conditional regulatory approval based on results from Phase II trials. However, if clinical benefit cannot be confirmed in larger post-marketing studies, marketing authorization and reimbursement should be revoked (e.g., recommendation from EMA to withdraw Lartruvo in April 2019 based on the results from the ANNOUNCE study [24]).

In short, a certain level of flexibility in regulatory and reimbursement decision-making may be needed to deal with exceptional circumstances, but by default, strict requirements and timelines should be laid down with regard to the provision of post-approval data that show clinical benefit. Clinical benefit scales, such as the ESMO MCBS, provide a framework to quantify the value of that benefit. The use of such scales in HTA and reimbursement decisions, and indeed regulatory approval, should be further encouraged. Interestingly, a joint assessment of the American Society of Oncology Value Framework and the ESMO MCBS has led to suggestions for potential approaches to increase convergence of the two frameworks [25].

\section{- Provide public funding \& support for noncommercial, independent trials}

Many interventions that could deliver meaningful clinical benefit to patients do not attract industry support. Such interventions include surgery, radiotherapy or new uses for existing, often generic, chemotherapy drugs and drug repurposing. Clinical innovation and improved survival can come from sources other than new molecules, but without improved funding for independent trials, such innovations may be stifled.

Innovative trial designs, like multi-arm/multi-stage or adaptive platform trials, may be worth exploring further, as such designs hold the promise of accelerating clinical development, reducing competition for patients and ensuring high-quality results. Moreover, in an independent trial infrastructure, both commercial and noncommercial treatments could be evaluated side-by-side in a patient-centered way [26-28].

\section{- Improve early contacts between clinicians, regulators \& HTA}

Early engagement with regulators is a standard feature of industry-led drug development, but less so for academics and clinicians. Early engagement through 'scientific advice' or other programs provides early feedback on trial design, including choice of end points, which may be instrumental in improving the quality of non-industry trials [29]. The EMA PRIME priority medicines program, which aims to facilitate the development of medicines 
targeting unmet medical needs, is one initiative to explore this option [30]. This program offers scientific advice and early dialogue between regulators and medicine developers to optimize benefit-risk data generation. Additionally, PRIME medicines are eligible for accelerated assessment at the time of marketing authorization application [31,32]. However, evidence suggests that uptake by academia is low and, therefore, efforts directed specifically at independent/academic investigators may be required to improve on this [29]. Similar initiatives to PRIME exist elsewhere, such as the breakthrough therapy designation program in the USA and the Sakigake Designation in Japan [33].

Increased early contacts and joint scientific advice with both regulators and HTA bodies should also be encouraged to identify the best comparator in clinical trials and facilitate the choice of primary end points that deliver added therapeutic value, which would ultimately accelerate access to new treatment options for cancer patients $[34,35]$.

\section{Conclusion}

The study by Naci et al. raises numerous questions relating to the design characteristics, risks of bias and reporting of pivotal clinical trials in oncology. These findings add to the existing body of evidence that suggests drug development in oncology is not delivering the clinical benefits that are required to meet the high unmet needs of cancer patients. Lowering the evidentiary bar increases the number of drug approvals but does not appear to significantly improve overall survival or provide meaningful clinical benefit. We have proposed a number of measures to address some of the points raised - but the topic warrants increased attention from clinicians, researchers, patients and policy-makers.

\section{Financial \& competing interests disclosure}

The authors have no relevant affiliations or financial involvement with any organization or entity with a financial interest in or financial conflict with the subject matter or materials discussed in the manuscript. This includes employment, consultancies, honoraria, stock ownership or options, expert testimony, grants or patents received or pending, or royalties.

No writing assistance was utilized in the production of this manuscript.

\section{References}

1. Naci H, Davis C, Savović J et al. Design characteristics, risk of bias, and reporting of randomised controlled trials supporting approvals of cancer drugs by European Medicines Agency, 2014-16: cross sectional analysis. BMJ 366, 15221 (2019).

2. Downing NS, Aminawung JA, Shah ND, Krumholz HM, Ross JS. Clinical trial evidence supporting FDA approval of novel therapeutic agents, 2005-2012. JAMA 311(4), 368-377 (2014).

3. Chen EY, Raghunathan V, Prasad V. An overview of cancer drugs approved by the US Food and Drug Administration based on the surrogate end point of response rate. JAMA Intern. Med. 179(7), 915-921 (2019).

4. Yothers G. Toward progression-free survival as a primary end point in advanced colorectal cancer. J. Clin. Oncol. 25(33), 5153-5154 (2007).

5. Kemp R, Prasad V. Surrogate endpoints in oncology: when are they acceptable for regulatory and clinical decisions, and are they currently overused? BMC Med. 15(1), 1-7 (2017).

6. Haslam A, Hey SP, Gill J, Prasad V. A systematic review of trial-level meta-analyses measuring the strength of association between surrogate end points and overall survival in oncology. Eur. J. Cancer 106, 196-211 (2019).

7. Kim C, Prasad V. Cancer drugs approved on the basis of a surrogate end point and subsequent overall survival: an analysis of 5 years of US Food and Drug Administration approvals. JAMA Intern. Med. 175(12), 1992-1994 (2015).

8. Davis C, Naci H, Gurpinar E, Poplavska E, Pinto A, Aggarwal A. Availability of evidence of benefits on overall survival and quality of life of cancer drugs approved by European Medicines Agency: retrospective cohort study of drug approvals 2009-13. BMJ359, j4530 (2017).

9. Pease AM, Krumholz HM, Downing NS, Aminawung JA, Shah ND, Ross JS. Postapproval studies of drugs initially approved by the FDA on the basis of limited evidence: systematic review. BMJ 357, j1680 (2017).

10. Cherny NI, Sullivan R, Dafni U et al. A standardised, generic, validated approach to stratify the magnitude of clinical benefit that can be anticipated from anti-cancer therapies: the European Society for Medical Oncology Magnitude of Clinical Benefit Scale (ESMO-MCBS). Ann. Oncol. 26(8), 1547-1573 (2015).

11. Schuller Y, Biegstraaten M, Hollak CEM, Klümpen H-J, Gispen-de Wied CC, Stoyanova-Beninska V. Oncologic orphan drugs approved in the EU - do clinical trial data correspond with real-world effectiveness? Orphanet J. Rare Dis. 13(1), 214 (2018).

12. Wild C, Grössmann N, Bonanno PV et al. Utilisation of the ESMO-MCBS in practice of HTA. Ann. Oncol. 27(11), $2134-2136$ (2016).

13. Grössmann N, Del Paggio JC, Wolf S et al. Five years of EMA-approved systemic cancer therapies for solid tumours-a comparison of two thresholds for meaningful clinical benefit. Eur. J. Cancer 82, 66-71 (2017).

14. Tibau A, Molto C, Ocana A et al. Magnitude of clinical benefit of cancer drugs approved by the US Food and Drug administration. J. Natl Cancer Inst. 110(5), 486-492 (2018). 
15. Saluja R, Arciero VS, Cheng $S$ et al. Examining trends in cost and clinical benefit of novel anticancer drugs over time. J. Oncol. Pract. 14(5), JOP1700058 (2018).

16. Vivot A, Jacot J, Zeitoun J-D, Ravaud P, Crequit P, Porcher R. Clinical benefit, price and approval characteristics of FDA-approved new drugs for treating advanced solid cancer, 2000-2015. Ann. Oncol. 28(5), 1111-1116 (2017).

17. Gyawali B, Bouche G, Pantziarka P, Kesselheim AS, Sarpatwari A. Lung cancer survival gains: contributions of academia and industry. J. Law. Med. Ethics 47(3), 465-467 (2019).

18. Saluja R, Arciero VS, Cheng S et al. Examining trends in cost and clinical benefit of novel anticancer drugs over time. J. Oncol. Pract. 14(5), JOP1700058 (2018).

19. Grössmann N, Del Paggio JC, Wolf S et al. Five years of EMA-approved systemic cancer therapies for solid tumours-a comparison of two thresholds for meaningful clinical benefit. Eur. J. Cancer 82, 66-71 (2017).

20. FDA.. FDA approves niraparib for HRD-positive advanced ovarian cancer. (2019). www.fda.gov/drugs/resources-information-approved-drugs/fda-approves-niraparib-hrd-positive-advanced-ovarian-cancer

21. Pantziarka P, Verbaanderd C, Sukhatme V et al. ReDO_DB: the repurposing drugs in oncology database. Ecancermedicalscience 12,886 (2018). https://ecancer.org/journal/12/full/886-redo_db-the-repurposing-drugs-in-oncology-database.php

22. Kim C, Prasad V. Cancer drugs approved on the basis of a surrogate end point and subsequent overall survival: an analysis of 5 years of US Food and Drug Administration approvals. JAMA Intern. Med. 175(12), 1992-1994 (2015).

23. Fojo T, Mailankody S, Lo A. Unintended consequences of expensive cancer therapeutics - the pursuit of marginal indications and a me-too mentality that stifles innovation and creativity: the John Conley lecture. JAMA Otolaryngol. Head Neck Surg. 140(12), 1225-1236 (2014).

24. Results reported from the Phase III ANNOUNCE trial of olaratumab in soft-tissue sarcoma - the ASCO post. www.ascopost.com/News/59688?utm_source=TrendMD\&utm_medium=cpc\&utm_campaign=Sarcoma_TrendMD_0

25. Cherny NI, de Vries EGE, Dafni U et al. Comparative assessment of clinical benefit using the ESMO-magnitude of clinical benefit scale version 1.1 and the ASCO value framework net health benefit score. J. Clin. Oncol. 37(4), 336-349 (2019).

26. The Adaptive Platform Trials Coalition.. Adaptive platform trials: definition, design, conduct and reporting considerations. Nat. Rev. Drug Discov. 18(10), 797-807 (2019).

27. Parmar MKB, Carpenter J, Sydes MR. More multiarm randomised trials of superiority are needed. Lancet 384(9940), 283-284 (2014).

28. Woodcock J, LaVange LM. Master protocols to study multiple therapies, multiple diseases, or both. N. Engl. J. Med. 377(1), 62-70 (2017).

29. Pantziarka P. Scientific advice - is drug repurposing missing a trick? Nat. Rev. Clin. Oncol. 14(8), 455-456 (2017).

30. EMA.. PRIME: priority medicines. (2019). www.ema.europa.eu/en/human-regulatory/research-development/prime-priority-medicines

31. Neez E, Hwang T, Sahoo SA, Naci H. European Medicines Agency's Priority Medicines (PRIME) scheme at 2years: an evaluation of clinical studies supporting eligible drugs. Clin. Pharmacol. Ther. doi.org/10.1002/cpt.1669 (2019). [Epub ahead of print]

32. Mullard A. PRIME time at the EMA. Nat. Rev. Drug Discov. 16(4), 226-228 (2017).

33. Kondo H, Hata T, Ito K, Koike H, Kono N. The current status of Sakigake designation in Japan, PRIME in the European Union, and Breakthrough Therapy Designation in the United States. Ther. Innov. Regul. Sci. 51(1), 51-54 (2017).

34. Tafuri G, Lucas I, Estevão $S$ et al. The impact of parallel regulatory-health technology assessment scientific advice on clinical development. Assessing the uptake of regulatory and health technology assessment recommendations. Br. J. Clin. Pharmacol. 84(5), 1013-1019 (2018).

35. Eichler H-G, Enzmann H, Rasi G. Added therapeutic benefit and drug licensing. Nat. Rev. Drug Discov. 18(9), 651-652 (2019). 
